\title{
$\operatorname{CSDT}(Q) / C B S$ thermochemistry for the $D_{5 h} \rightarrow D_{10 h}$ isomerization
}

\section{in the $\mathrm{C}_{10}$ carbon cluster: Getting the right answer for the right}

\author{
reason \\ Amir Karton $^{\dagger, *}$ and Venkatesan S. Thimmakondu* \\ ${ }^{\dagger}$ School of Molecular Sciences, The University of Western Australia, Perth, WA 6009, \\ Australia. \\ ${ }^{\ddagger}$ Department of Chemistry, Birla Institute of Technology and Science, Pilani, K K Birla Goa \\ Campus, Goa, 403726, India.
}

\begin{abstract}
.
The $\mathrm{D}_{5 \mathrm{~h}} \rightarrow \mathrm{D}_{10 \mathrm{~h}}$ isomerization in the $\mathrm{C}_{10}$ carbon cluster is investigated at the relativistic, allelectron CCSDT(Q)/CBS level. Previous high-level studies examined this isomerization at the valence $\operatorname{CCSD}(\mathrm{T}) / \mathrm{CBS}$ level. We show that capturing this isomerization energy requires accurate treatment of the $\operatorname{CCSD}(\mathrm{T}) / \mathrm{CBS}$, post-CCSD $(\mathrm{T})$, core-valence, scalar relativistic, diagonal Born-Oppenheimer, and zero-point vibrational energy components. Combining these components shows that the two structures are practically isoenergetic at $0 \mathrm{~K}$ (i.e., the $\mathrm{D}_{5 \mathrm{~h}}$ structure is more stable by merely $+0.100 \mathrm{kcal} \mathrm{mol}^{-1}$ ). We also show that computationally economical composite protocols erroneously predict that the $\mathrm{D}_{10 \mathrm{~h}}$ structure is energetically more stable at $0 \mathrm{~K}$.
\end{abstract}




\section{Introduction}

Small carbon clusters have attracted considerable interest due to their importance in interstellar ${ }^{1,2}$ and combustion chemistry, ${ }^{3,4}$ and as potential precursors of fullerenes, ${ }^{5,6}$ carbon nanotubes, ${ }^{7,8,9}$ grapheme, ${ }^{10,11}$ and thin diamond films generated by chemical vapor deposition. ${ }^{12}$ Carbon clusters with an even number of carbons can exist in both linear (triplet ${ }^{3} \square \sum_{\mathrm{g}}$ ) and cyclic (singlet ${ }^{1} \mathrm{~A}$ ) forms with comparable energies. ${ }^{12}$ Where $\mathrm{C}_{10}$ is the smallest carbon cluster for which the cyclic structure is more stable than the linear structure. This is attributed to aromatic stabilization effects associated with in-plane and perpendicular aromatic systems in $\mathrm{C}_{10}$ and to a lower strain energy compared with smaller cyclic carbon clusters. ${ }^{12}$ For this reason, the structure of the cyclic $\mathrm{C}_{10}$ cluster has attracted considerable attention over the past four decades. ${ }^{12,13,14,15,16,17,18,19,20,21,22,23}$ It has long been thought that the cumulenic $\mathrm{D}_{5 \mathrm{~h}}$ (equal bonds, alternating angles) structure is a local minimum and the regular decagon $\mathrm{D}_{10 \mathrm{~h}}$ structure is a first-order saddle point. ${ }^{15,17}$ However, the energy difference between the two structures has been a matter of some controversy. ${ }^{14,15,17,20,21}$

Nearly 40 years ago Liang and Schaefer calculated this energy difference using configuration interaction with singles and doubles (CISD) in conjunction with a small double- $\zeta$ basis set. They found that the $\mathrm{D}_{5 \mathrm{~h}}$ structure is more stable than the $\mathrm{D}_{10 \mathrm{~h}}$ structure by as much as $18.5 \mathrm{kcal} \mathrm{mol}^{-1}$. Applying a Davidson correction for unlinked diagrams reduces this difference to $12.9 \mathrm{kcal} \mathrm{mol}^{-1}$. However, they noted that due to the sensitivity of this energy difference to higher-order correlation effects it would be premature to conclude that the $D_{5 h}$ structure is more stable. Shortly thereafter, Watts and Bartlett calculated the $D_{5 h}$ structure to be more stable by a significantly smaller amount of just $0.8 \mathrm{kcal} \mathrm{mol}^{-1}$ using coupled cluster theory with singles, doubles, and quasiperturbative triple excitations $(\operatorname{CCSD}(\mathrm{T}))$ in conjunction with a larger double- $\zeta$ basis set. Martin and Taylor also concluded that the $\mathrm{D}_{5 \mathrm{~h}}$ structure is more stable, ${ }^{17}$ and estimated an energy difference of $1.0 \pm 0.1 \mathrm{kcal}$ 
$\mathrm{mol}^{-1}$ at the $\operatorname{CCSD}(\mathrm{T}) / \mathrm{cc}-\mathrm{pVTZ}$ level of theory. Yousaf and Taylor calculated the $\mathrm{D}_{5 \mathrm{~h}}$ structure to be more stable by merely $0.2 \mathrm{kcal} \mathrm{mol}^{-1}$ at the $\operatorname{CCSD}(\mathrm{T})$ level in conjunction with a $4 \mathrm{~s} 3 \mathrm{p} 2 \mathrm{~d}$ atomic natural orbital (ANO) basis set. ${ }^{18}$ Finally, Karton and Martin calculated the reaction barrier height at the $\operatorname{CCSD}(\mathrm{T}) / \mathrm{cc}-\mathrm{pVTZ}$ and $\operatorname{CCSD}(\mathrm{T}) / \mathrm{cc}-\mathrm{pVQZ}$ levels of theory and obtained barriers of 1.11 and $0.33 \mathrm{kcal} \mathrm{mol}^{-1}$, respectively. ${ }^{21}$ Extrapolating the $\operatorname{CCSD}(\mathrm{T})$ energy from the cc-pV\{Q,5\}Z basis-set pair resulted in a barrier of $0.30 \mathrm{kcal} \mathrm{mol}^{-1}$. These studies indicate that at the complete basis set (CBS) frozen-core, non-relativistic, clamped nuclei $\operatorname{CCSD}(\mathrm{T})$ level the energy separation between the two structures should be about $1 \mathrm{~kJ}$ $\mathrm{mol}^{-1}$. Nevertheless, it is reasonable to assume that secondary energetic contributions (such as core-valence, scalar relativistic, zero-point vibrational energy, and deviations from the BornOppenheimer approximation) will have contributions on the order of $\sim 1 \mathrm{~kJ} \mathrm{~mol}^{-1}$ (or larger) to the isomerization energy. In addition, the $\mathrm{C}_{10}$ clusters involve an appreciable degree of multireference character and therefore post-CCSD(T) contributions are expected to affect the isomerization energy by more than $1 \mathrm{~kJ} \mathrm{~mol}^{-1}$. For example, the $\mathrm{D}_{5 \mathrm{~h}}$ and $\mathrm{D}_{10 \mathrm{~h}}$ structures are associated with \% TAE[(T)] diagnostics of $4.7 \%$ and $5.0 \%$, respectively (calculated in conjunction with the cc-pVTZ basis set). These diagnostics are defined as: \% TAE[(T)] = $100 \times(\mathrm{TAE}[\mathrm{CCSD}(\mathrm{T})]-\mathrm{TAE}[\mathrm{CCSD}])$ / TAE[CCSD(T)], where TAE[CCSD] and $\operatorname{TAE}[\operatorname{CCSD}(\mathrm{T})]$ are the total atomization energies calculated at the CCSD and $\operatorname{CCSD}(\mathrm{T})$ levels, respectively. ${ }^{24}$ This energy-based diagnostic has been found to be a good a priori diagnostic for the importance of post-CCSD(T) contributions. ${ }^{24,25,26}$

In the present work we consider both secondary energetic contributions (namely, core-valence, scalar relativistic, zero-point vibrational energy, and deviations from the BornOppenheimer approximation) and post-CCSD(T) contributions to the isomerization energy. We show that both post-CCSD(T) and ZPVE contributions to the isomerization energy far exceed $1 \mathrm{kcal} \mathrm{mol}^{-1}$ and accurate treatment of all the above-mentioned components is 
essential for determining the barrier with benchmark accuracy (i.e., to within $\sim 1 \mathrm{~kJ} \mathrm{~mol}^{-1}$ ).

\section{Computational details}

The geometries of all structures were obtained at the CCSD(T)/cc-pVQZ level of theory and taken from ref. 21. The CCSDT(Q)/CBS energies have been calculated by means of the W4lite computational thermochemistry protocol $^{24}$ (with the exception of the $\mathrm{T}-(\mathrm{T})$ component which is taken from W3.2lite theory). ${ }^{27}$ The basis-set extrapolations involved in W4lite and W3.2lite theories have been specified and rationalized in great detail in refs. 24 and 27, respectively (see also ref. 25 for a recent review of these theories).

All the large-scale $\operatorname{CCSD}(\mathrm{T})$ calculations were performed using the Molpro program suite, ${ }^{28,29}$ the post-CCSD(T) calculations were performed with the MRCC program, ${ }^{30,31}$ the diagonal Born-Oppenheimer corrections (DBOC) and harmonic zero-point vibrational energy (ZPVE) calculations were carried out with the CFOUR program, ${ }^{32}$ and anharmonic ZPVE calculations were performed with the Gaussian 09 program. ${ }^{33}$

For the large-scale SCF and coupled cluster calculations, we employed the cc-pVnZ basis sets. ${ }^{34}$ In the core-valence $\operatorname{CCSD}(\mathrm{T})$ calculations, the core-valence weighted correlation consistent basis sets of Peterson and Dunning were employed. ${ }^{35}$ Scalar relativistic CCSD(T) calculations were carried out using the Pacific Northwest National Laboratory (PNNL) Douglas-Kroll-Hess relativistically contracted correlation basis sets. ${ }^{36}$

\section{Results and discussion}

3.1. The self-consistent field (SCF) energy. Table 1 gives an overview of the basis set convergence of the SCF component of the $\mathrm{D}_{5 \mathrm{~h}} \rightarrow \mathrm{D}_{10 \mathrm{~h}}$ isomerization energy. Our best CBS SCF component of $23.866 \mathrm{kcal} \mathrm{mol}^{-1}$ is extrapolated from the cc-pV5Z and cc-pV6Z basis sets using the Karton-Martin ${ }^{37}$ modification of Jensen's extrapolation formula. ${ }^{38}$ Calculating 
the SCF energy in conjunction with the cc-pVDZ basis set results in an overestimation of 1.3 $\mathrm{kcal} \mathrm{mol}^{-1}$, relative to the CBS value. This error drops to $0.3 \mathrm{kcal} \mathrm{mol}^{-1}$ when the cc-pVTZ basis set is used. Extrapolation of the SCF energy from the cc-pVDZ and cc-pVTZ basis sets using the Karton-Martin basis-set extrapolation still results in an error of $0.2 \mathrm{kcal} \mathrm{mol}^{-1}$. These errors are certainly too large for the purpose of the current investigation. Calculating the SCF energy in conjunction with the cc-pVQZ basis set results in an overestimation of the SCF component by $0.04 \mathrm{kcal} \mathrm{mol}^{-1}$ and extrapolating from the cc-pVTZ and cc-pVQZ basis sets reduces this error to $0.01 \mathrm{kcal} \mathrm{mol}^{-1}$. We can therefore conclude that the SCF component is nearly converged at the cc-pV\{T,Q $\} Z$ level. Extrapolation from the cc-pVQZ and cc-pV5Z basis sets results in a near-zero error of $0.001 \mathrm{kcal} \mathrm{mol}^{-1}$, relative to the $\mathrm{CBS}$ value.

Table 1. Overview of the basis set convergence of the SCF component of the $D_{5 h} \rightarrow D_{10 h}$ isomerization energy $\left(\mathrm{kcal} \mathrm{mol}^{-1}\right)$

$\begin{array}{lc}\text { Basis sets } & \text { SCF } \\ \text { cc-pVDZ } & 25.123 \\ \text { cc-pVTZ } & 24.154 \\ \text { cc-pVQZ } & 23.911 \\ \text { cc-pV5Z } & 23.874 \\ \text { cc-pV6Z } & 23.867 \\ \text { cc-pV }\{\mathrm{D}, \mathrm{T}\} Z^{a} & 24.007 \\ \text { cc-pV }\{\mathrm{T}, \mathrm{Q}\} Z^{a} & 23.835 \\ \text { cc-pV }\{\mathrm{Q}, 5\} Z^{a} & 23.856 \\ \text { cc-pV }\{5,6\} Z^{a} & 23.863 \\ \text { cc-pV }\{\mathrm{D}, \mathrm{T}\} Z^{b} & 24.074 \\ \text { cc-pV }\{\mathrm{T}, \mathrm{Q}\} Z^{b} & 23.880 \\ \text { cc-pV }\{\mathrm{Q}, 5\} Z^{b} & 23.867 \\ \text { cc-pV }\{5,6\} Z^{b} & 23.866\end{array}$

${ }^{a}$ Extrapolated using the $\mathrm{E}(L)=\mathrm{E}_{\infty}+\mathrm{A} / L^{5}$ two-point extrapolation.

${ }^{b}$ Extrapolated using the Karton-Martin extrapolation. ${ }^{37}$

It is of interest to compare the CBS limits obtained using the $\mathrm{E}(L)=\mathrm{E}_{\infty}+\mathrm{A} / L^{5}$ twopoint extrapolation formula with those obtained using the Karton-Martin extrapolation. These results are depicted in Table 1 . When the cc-pV $\{\mathrm{D}, \mathrm{T}\} \mathrm{Z}$ basis set pair is used the 
difference between the two extrapolations is fairly large and amounts to $0.07 \mathrm{kcal} \mathrm{mol}^{-1}$. This difference drops to 0.04 and $0.01 \mathrm{kcal} \mathrm{mol}^{-1}$, respectively for the cc-pV\{T,Q\}Z and cc$\mathrm{pV}\{\mathrm{Q}, 5\} \mathrm{Z}$ basis set pairs, respectively. When the cc-pV $\{5,6\} \mathrm{Z}$ basis set pair is used the two extrapolations result in essentially the same CBS value (namely, 23.863 and $23.866 \mathrm{kcal} \mathrm{mol}^{-}$ ${ }^{1}$, respectively). The difference between these two CBS estimates $\left(0.003 \mathrm{kcal} \mathrm{mol}^{-1}\right)$ can be taken as the uncertainty in our best SCF isomerization energy.

3.2. The valence CCSD correlation energy. Table 2 gives an overview of the basis set convergence of the CCSD component of the $\mathrm{D}_{5 \mathrm{~h}} \rightarrow \mathrm{D}_{10 \mathrm{~h}}$ isomerization energy. Our best CBS CCSD component of $-19.099 \mathrm{kcal} \mathrm{mol}^{-1}$ is extrapolated from the cc-pV5Z and cc-pV6Z basis sets using the extrapolation formula from W4 theory, ${ }^{24,39,40}$ in which the singlet-coupled and triplet-coupled pair energies are extrapolated separately to the CBS limit. Interestingly, the cc-pVnZ basis sets $(n=2-6)$ do not converge monotonically to the CBS limit. In particular, the following errors are obtained relative to our best CBS value: -0.056 (cc-pVDZ), -0.013

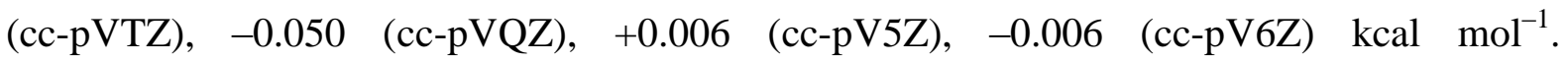
Accordingly, the cc-pV $\{n-1, n\} \mathrm{Z}$ extrapolations $(n=3-6)$ do not converge smoothly to the CBS limit either. For example, the following errors are obtained relative to our best CBS value: +0.006 (cc-pV\{D,T\}Z), $-0.077(\mathrm{cc}-\mathrm{pV}\{\mathrm{T}, \mathrm{Q}\} \mathrm{Z})$, and +0.064 (cc-pV\{Q,5\}Z) kcal mol ${ }^{-}$ ${ }^{1}$. Due to this oscillatory basis-set convergence we will assign a (conservative) estimated uncertainty of $0.05 \mathrm{kcal} \mathrm{mol}^{-1}$ to our best $\mathrm{CCSD} / \mathrm{cc}-\mathrm{pV}\{5,6\} \mathrm{Z}$ energy. 
Table 2. Overview of the valence CCSD correlation contributions to the $D_{5 h} \rightarrow D_{10 h}$ isomerization energy $\left(\mathrm{kcal} \mathrm{mol}^{-1}\right)$

$\begin{array}{lc}\text { Basis sets } & \text { CCSD } \\ \text { cc-pVDZ } & -19.155 \\ \text { cc-pVTZ } & -19.112 \\ \text { cc-pVQZ } & -19.149 \\ \text { cc-pV5Z } & -19.093 \\ \text { cc-pV6Z } & -19.105 \\ \text { cc-pV }\{\mathrm{D}, \mathrm{T}\} Z^{a} & -19.093 \\ \text { cc-pV }\{\mathrm{T}, \mathrm{Q}\} Z^{a} & -19.176 \\ \text { cc-pV }\{\mathrm{T}, \mathrm{Q}\} Z^{b} & -19.173 \\ \text { cc-pV }\{\mathrm{Q}, 5\} Z^{a} & -19.035 \\ \text { cc-pV }\{\mathrm{Q}, 5\} Z^{c} & -19.011 \\ \text { cc-pV }\{5,6\} Z^{a} & -19.121 \\ \text { cc-pV }\{5,6\} Z^{d} & -19.099\end{array}$

${ }^{a}$ The CCSD basis-set limits are extrapolated using $\mathrm{E}(L)=\mathrm{E}_{\infty}+\mathrm{A} / L^{3}$ two-point extrapolation formula.

${ }^{b}$ Extrapolated using $\mathrm{E}(L)=\mathrm{E}_{\infty}+\mathrm{A} / L^{3.22}$ two-point extrapolation formula from W1 theory.

${ }^{c} \mathrm{CBS}$ limit value from W2.2 theory. ${ }^{41,42}$

${ }^{d}$ Best CBS limit value from W4 theory. ${ }^{24,39,40}$

3.3. The valence (T) correlation energy. Table 3 gives an overview of the basis set convergence of the $(T)$ component of the $\mathrm{D}_{5 \mathrm{~h}} \rightarrow \mathrm{D}_{10 \mathrm{~h}}$ isomerization energy. Our best CBS value of $-4.518 \mathrm{kcal} \mathrm{mol}^{-1}$ is extrapolated from the cc-pVQZ and cc-pV5Z basis sets as prescribed in W4 theory. ${ }^{24}$ In contrast to the CCSD component (see above), the (T) correlation component converges fairly smoothly to the CBS limit. In particular, we obtain errors of 1.205 (cc-pVDZ), 0.256 (cc-pVTZ), 0.089 (cc-pVQZ), and 0.045 (cc-pV5Z) kcal $\mathrm{mol}^{-1}$. However, it is evident that the convergence to the CBS is rather slow. The cc$\mathrm{pV}\{\mathrm{D}, \mathrm{T}\} \mathrm{Z}$ extrapolation results in a fairly large error of $-0.144 \mathrm{kcal} \mathrm{mol}^{-1}$. This error is reduced to an acceptable value of $-0.032 \mathrm{kcal} \mathrm{mol}^{-1}$ for the cc-pV\{T,Q\}Z extrapolation. 
Table 3. Overview of the valence $(T)$ correlation contributions to the $D_{5 h} \rightarrow D_{10 h}$ isomerization energy $\left(\mathrm{kcal} \mathrm{mol}^{-1}\right)$

$\begin{array}{lc}\text { Basis sets } & (\mathbf{T}) \\ \text { cc-pVDZ } & -3.313 \\ \text { cc-pVTZ } & -4.262 \\ \text { cc-pVQZ } & -4.429 \\ \text { cc-pV5Z } & -4.473 \\ \text { cc-pV }\{\mathrm{D}, \mathrm{T}\} Z^{a} & -4.662 \\ \text { cc-pV }\{\mathrm{T}, \mathrm{Q}\} Z^{a} & -4.550 \\ \text { cc-pV }\{\mathrm{Q}, 5\} Z^{a} & -4.518\end{array}$

${ }^{a}$ The $(\mathrm{T})$ basis-set limits are extrapolated using $\mathrm{E}(L)=\mathrm{E}_{\infty}+\mathrm{A} / L^{3}$ two-point extrapolation formula.

3.4. The all-electron CCSD(T) correlation energy. The above results suggest that the valence $\operatorname{CCSD}(\mathrm{T})$ energy converges fairly slowly to the CBS limit. Table 4 gives the breakdown of the valence $\operatorname{CCSD}(\mathrm{T})$ isomerization energy from $\mathrm{W} 1, \mathrm{~W} 2.2$, and $\mathrm{W} 4$ theories. Overall, all three theories predict that the $\mathrm{D}_{5 \mathrm{~h}}$ structure is slightly more stable than the $\mathrm{D}_{10 \mathrm{~h}}$ structure. However, whilst at the W4 level the energy difference between the two structures is $0.25 \mathrm{kcal} \mathrm{mol}^{-1}$, this difference is slightly overestimated by W2.2 theory and significantly underestimated by W1 theory, which predicts an energy separation of just $0.05 \mathrm{kcal} \mathrm{mol}^{-1}$.

Table 4. Breakdown of the valence and all-electron CCSD(T)/CBS $\mathrm{D}_{5 \mathrm{~h}} \rightarrow \mathrm{D}_{10 \mathrm{~h}}$ isomerization energies from W1, W2.2, and W4 theories $\left(\mathrm{kcal} \mathrm{mol}^{-1}\right)$

$\begin{array}{lccccc}\text { Theory } & \text { SCF } & \text { Val. CCSD } & \text { Val. (T) } & \text { Val. CCSD(T) } & \text { All-electron CCSD(T) } \\ \text { W1 } & 23.835 & -19.173 & -4.615 & 0.047 & -0.213 \\ \text { W2.2 } & 23.867 & -19.011 & -4.550 & 0.306 & 0.046 \\ \text { W4 } & 23.866 & -19.099 & -4.518 & 0.248 & -0.012\end{array}$

Let us turn our attention to core-valence $(\mathrm{CV})$ effects. It turns out that the $\mathrm{CV}$ component stabilizes the $\mathrm{D}_{10 \mathrm{~h}}$ structure to a larger extent than the $\mathrm{D}_{5 \mathrm{~h}}$ structure. Namely, the $\mathrm{CV}$ component for the $\mathrm{D}_{5 \mathrm{~h}} \rightarrow \mathrm{D}_{10 \mathrm{~h}}$ isomerization amounts to -0.233 and $-0.249 \mathrm{kcal} \mathrm{mol}^{-1}$ at the $\operatorname{CCSD}(\mathrm{T}) / \mathrm{cc}-\mathrm{pwCVTZ}$ and $\operatorname{CCSD}(\mathrm{T}) / \mathrm{cc}-\mathrm{pwCVQZ}$ levels of theory, respectively. At the 
$\operatorname{CCSD}(\mathrm{T}) / \mathrm{cc}-\mathrm{pwCV}\{\mathrm{T}, \mathrm{Q}\} \mathrm{Z}$ level of theory the CV contribution amounts to $-0.260 \mathrm{kcal} \mathrm{mol}^{-}$ 1

Thus, the CCSD(T)/CBS CV component, which stabilizes the $\mathrm{D}_{10 \mathrm{~h}}$ isomer by 0.260 $\mathrm{kcal} \mathrm{mol}^{-1}$, cancels out almost perfectly with the valence $\operatorname{CCSD}(\mathrm{T}) / \mathrm{CBS}$ component, which stabilizes the $\mathrm{D}_{5 \mathrm{~h}}$ isomer by $0.248 \mathrm{kcal} \mathrm{mol}^{-1}$ (at the W4 level). Combining these two components results in a near-zero all-electron $\operatorname{CCSD}(\mathrm{T}) / \mathrm{CBS}$ isomerization energy of -0.012 $\mathrm{kcal} \mathrm{mol}^{-1}$ at the $\mathrm{W} 4$ level. In this situation, it is of interest to examine the magnitude of post$\operatorname{CCSD}(\mathrm{T})$ correlation effects as well as other corrections (such as scalar relativistic and diagonal Born-Oppenheimer corrections).

3.5. CCSDT and CCSDT(Q) correlation effects. Table 5 gives an overview of the post$\operatorname{CCSD}(\mathrm{T})$ correlation components. We were able to calculate the CCSDT-CCSD(T) component in conjunction with the cc-pVDZ and cc-pVTZ(no f 1d) basis sets, where ccpVTZ(no $\mathrm{f} 1 \mathrm{~d}$ ) is a truncated version of the cc-pVTZ basis set which combines the sp part of the cc-pVTZ basis set with the d function from the cc-pVDZ basis set. We note that the CCSDT/cc-pVTZ(no $f$ 1d) calculation for the $\mathrm{D}_{5 \mathrm{~h}}$ isomer was especially demanding computationally, requiring 3.5 billion amplitudes and taking approximately one week on 20 Intel Xeon $2.00 \mathrm{GHz}$ cores with $256 \mathrm{~GB}$ of random-access memory (RAM). With the ccpVDZ and cc-pVTZ(no f 1d) basis sets we obtain a T-(T) component of 1.81 and $1.98 \mathrm{kcal}$ $\mathrm{mol}^{-1}$, respectively. Extrapolating these two values using the extrapolation formula prescribed in ref. 27 results in our best $\mathrm{T}-(\mathrm{T})$ value of $2.24 \mathrm{kcal} \mathrm{mol}^{-1}$. The quasiperturbative connected quadruple excitations were calculated in conjunction with the cc-pVDZ basis set and the (Q) component amounts to only $-0.10 \mathrm{kcal} \mathrm{mol}^{-1}$. Overall, post-CCSD(T) contributions stabilize the $D_{5 \mathrm{~h}}$ structure by as much as $2.14 \mathrm{kcal} \mathrm{mol}^{-1}$ relative to the $\mathrm{D}_{10 \mathrm{~h}}$ isomer. This energy 
stabilization is nearly an order of magnitude larger than the energy stabilization calculated at the $\mathrm{CCSD}(\mathrm{T}) / \mathrm{CBS}$ level of theory (taken from W4 theory).

Table 5. Overview of the post-CCSD(T) correlation contributions to the $D_{5 h} \rightarrow D_{10 h}$ isomerization energy $\left(\mathrm{kcal} \mathrm{mol}^{-1}\right)$

$\begin{array}{lcc}\text { Basis sets } & \text { T-(T) } & (\mathbf{Q}) \\ \text { cc-pVDZ } & 1.81 & -0.10 \\ \text { cc-pVTZ(no f 1d) } & 1.98 & \text { N/A } \\ \text { cc-pV }\{\mathrm{D}, \mathrm{T}\} Z^{a} & 2.24 & \text { N/A }\end{array}$

${ }^{a}$ The $\mathrm{T}-(\mathrm{T})$ basis-set limits are extrapolated using a two-point extrapolation which is not based on the highest angular momentum (for further details see ref. 27).

3.6. Scalar relativistic, diagonal Born-Oppenheimer, and ZPVE corrections. So far we have considered the all-electron $\operatorname{CCSDT}(\mathrm{Q}) / \mathrm{CBS} \mathrm{D}_{5 \mathrm{~h}} \rightarrow \mathrm{D}_{10 \mathrm{~h}}$ isomerization energy. In this subsection we will calculate the scalar-relativistic and diagonal Born-Oppenheimer corrections. These results are presented in Table 6. The Scalar relativistic component stabilizes the $\mathrm{D}_{5 \mathrm{~h}}$ structure by an appreciable amount of $0.19 \mathrm{kcal} \mathrm{mol}^{-1}$ at the $\mathrm{CCSD}(\mathrm{T}) / \mathrm{cc}-$ pVQZ-DK level of theory.

The diagonal Born-Oppenheimer correction amounts to as much as $0.365 \mathrm{kcal} \mathrm{mol}^{-1}$ at the HF/cc-pVDZ level of theory. Using the larger cc-pVTZ basis set has practically no effect on the DBOC (Table 6). However, inclusion of correlation effects at the CCSD level reduces the DBOC component by an order of magnitude to just 0.036 (cc-pVDZ) and 0.040 (cc-pVTZ) kcal mol ${ }^{-1}$. Previous studies that considered organic systems of similar sizes have reported sizable differences between the $\mathrm{HF}$ and CCSD $\triangle \mathrm{DBOC}$ components. In particular, the correlation contributions to the DBOC can reduce the $\triangle \mathrm{DBOC}$ contribution by up to $50 \%$ for total atomization energies (e.g., see refs. 25 and 43, and refs. therein, for further discussion). The $\mathrm{D}_{5 \mathrm{~h}} \rightarrow \mathrm{D}_{10 \mathrm{~h}}$ isomerization represents an extreme case where the correlation 
contribution reduces the $\triangle \mathrm{DBOC}$ component by an order of magnitude, ostensibly due to the sensitivity of these systems to higher-order correlation effects.

Table 6. Overview of scalar-relativistic, diagonal Born-Oppenheimer, and ZPVE corrections to the $\mathrm{D}_{5 \mathrm{~h}} \rightarrow \mathrm{D}_{10 \mathrm{~h}}$ isomerization energy $\left(\mathrm{kcal} \mathrm{mol}^{-1}\right)$

$\begin{array}{llc}\text { Component } & \text { Level of theory } & \Delta E\left(\mathrm{D}_{5 \mathrm{~h}} \rightarrow \mathrm{D}_{10 \mathrm{~h}}\right) \\ \text { Scalar Relativistic } & \text { CCSD(T)/cc-pVQZ-DK } & 0.191 \\ \text { DBOC } & \text { HF/cc-pVDZ } & 0.365 \\ & \text { HF/cc-pVTZ } & 0.367 \\ & \text { CCSD/cc-pVDZ } & 0.036 \\ \text { Harmonic ZPVE } & \text { CCSD/cc-pVTZ } & 0.040 \\ \text { Harmonic ZPVE } & \text { CCSD(T)/cc-pVTZ } & -3.050 \\ \text { Anharmonic ZPVE } & a & -2.448 \\ \text { Anharmonic ZPVE } & b & -2.416 \\ \text { DD(T)/cc-pVTZ ZPVE scaled by } 0.9868 \text { as recommended in ref. } 44 . \\ \text { SD(T)/cc-pVTZ ZPVE combined with an anharmonic correction calculated at the B3LYP/pc-1 }\end{array}$

Table 7 summarizes our best components of the $D_{5 h} \rightarrow D_{10 h}$ isomerization energy. Summing up our best all-electron CCSDT(Q) isomerization energy $\left(2.131 \mathrm{kcal} \mathrm{mol}^{-1}\right)$ with the scalar relativistic $\left(0.191 \mathrm{kcal} \mathrm{mol}^{-1}\right)$ and DBOC $\left(0.040 \mathrm{kcal} \mathrm{mol}^{-1}\right)$ results in an allelectron, relativistic, DBOC-inclusive CCSDT(Q)/CBS isomerization energy of $2.362 \mathrm{kcal}$ $\mathrm{mol}^{-1}$. To convert this 'bottom-of-the-well' isomerization energy to an isomerization energy at $0 \mathrm{~K}$ we need to include the zero-point vibrational energy (ZPVE). We were able to calculate the harmonic ZPVE at the CCSD(T)/cc-pVTZ level of theory. At this level of theory, we obtain a ZPVE correction of $-2.448 \mathrm{kcal} \mathrm{mol}^{-1}$, i.e., the ZPVE corrections stabilizes the $\mathrm{D}_{10 \mathrm{~h}}$ isomer to a considerable amount. The most straightforward way to convert this harmonic ZPVE into an anharmonic ZPVE is to scale the $\operatorname{CCSD}(\mathrm{T})$ harmonic frequencies by an empirical scaling factor of 0.9868 as recommended in ref. 44 . This results in an anharmonic ZPVE of $-2.416 \mathrm{kcal} \mathrm{mol}^{-1}$. Calculating the cubic and quartic anharmonic corrections at the B3LYP/pc-1 level of theory ${ }^{45,46}$ results in a slightly smaller anharmonic 
ZPVE of $-2.262 \mathrm{kcal} \mathrm{mol}^{-1}$. Combining our best anharmonic ZPVE correction with our best 'bottom-of-the-well' all-electron, relativistic, DBOC-inclusive CCSDT(Q)/CBS isomerization energy results in a near-zero isomerization energy of $+0.100 \mathrm{kcal} \mathrm{mol}^{-1}$, indicating that the two structures are practically isoenergetic at $0 \mathrm{~K}$.

Table 7. Overview of our best valence $\operatorname{CCSD}(\mathrm{T})$, core-valence, post-CCSD(T), scalarrelativistic, diagonal Born-Oppenheimer, and ZPVE components of the $D_{5 h} \rightarrow D_{10 h}$ isomerization energy $\left(\mathrm{kcal} \mathrm{mol}^{-1}\right)$

$\begin{array}{lcc}\text { Component } & \text { Theory } & \Delta E\left(\mathrm{D}_{5 \mathrm{~h}} \rightarrow \mathrm{D}_{10 \mathrm{~h}}\right) \\ \text { Val. CCSD }(\mathrm{T}) & \text { W4lite } & +0.248 \\ \text { Core-valence } & \text { W4lite } & -0.260 \\ \text { Post-CCSD(T) } & \text { W3.2lite } & +2.143 \\ \text { Scalar Relativistic } & \text { W4lite } & +0.191 \\ \text { DBOC } & \text { W4.4 } & +0.040 \\ \text { Anharmonic ZPVE } & & -2.262 \\ \Delta H_{0} & & +0.100\end{array}$

Finally, a word is due regarding the estimated uncertainty in our best isomerization energy. In the current work, nearly all the electronic components are calculated by means of W4lite theory. ${ }^{24}$ The two exceptions are (i) the $\mathrm{T}-(\mathrm{T})$ component which was taken from W3.2lite theory, ${ }^{27}$ and (ii) the DBOC component which is calculated at the CCSD/cc-pVTZ level of theory rather than at the HF/aug-cc-pVTZ level. The $95 \%$ confidence interval (CI) of W4lite theory for total atomization energies (TAEs) for a set 18 first-row experimental TAEs associated with error bars $\leq 0.06 \mathrm{kcal} \mathrm{mol}^{-1}$ is $0.180 \mathrm{kcal} \mathrm{mol}^{-1} \cdot{ }^{25,47}$ This $95 \% \mathrm{CI}$ represents an upper limit since it is obtained for TAEs. The $D_{5 h} \rightarrow D_{10 h}$ isomerization, on the other hand, benefits from a large degree of systematic error cancellation between reactants and products, which are structurally and electronically similar to one another. ${ }^{21,26,27}$ Therefore, the uncertainty associated with the W4lite $D_{5 h} \rightarrow D_{10 h}$ isomerization energy should be significantly smaller than $0.180 \mathrm{kcal} \mathrm{mol}^{-1}$. It should be pointed out that uncertainties caused 
by issues with the harmonic ZPVE and the anharmonic correction to the ZPVE are expected to exceed those associated with the electronic structure treatment. The overall uncertainty is somewhat difficult to quantify, but a semi-quantitative estimate would probably be around the magnitude of the isomerization energy itself.

As pointed out in the introduction, previous studies that calculated the isomerization energy at the valence $\operatorname{CCSD}(\mathrm{T}) / \mathrm{CBS}$ level of theory have also reached the conclusion that the energy separation between the two structures is very small. For example, ref. 21 extrapolated the valence $\operatorname{CCSD}(T)$ from the $c c-p\{Q, 5\} Z$ basis set pair and obtained an isomerization energy of $+0.30 \mathrm{kcal} \mathrm{mol}^{-1}$. In the present work we obtain an isomerization energy of +0.25 $\mathrm{kcal} \mathrm{mol}^{-1}$ at the valence $\operatorname{CCSD}(\mathrm{T}) / \mathrm{cc}-\mathrm{p}\{5,6\} \mathrm{Z}$ level of theory. These results are not very far from our best all-electron, relativistic, DBOC-inclusive CCSDT(Q)/CBS isomerization energy at $0 \mathrm{~K}$ of $+0.10 \mathrm{kcal} \mathrm{mol}^{-1}$. We show that this fairly good agreement is due to a large cancellation between the post-CCSD(T) contribution (+2.143) and the ZPVE contribution ($\left.2.262 \mathrm{kcal} \mathrm{mol}^{-1}\right)$.

Finally, we note that computationally economical composite protocols that approximate the $\operatorname{CCSD}(\mathrm{T})$ energy at various degrees of basis set completeness (such as G4(MP2), ${ }^{48}$ G4(MP2)-6X, ${ }^{49} \mathrm{G} 4,{ }^{48}$ and CBS-QB3) ${ }^{50}$ are unable to predict the sign of the $\mathrm{D}_{5 \mathrm{~h}}$ $\rightarrow \mathrm{D}_{10 \mathrm{~h}}$ isomerization reaction. In particular, they predict that the $\mathrm{D}_{10 \mathrm{~h}}$ structure is energetically more stable than the $\mathrm{D}_{5 \mathrm{~h}}$ structure by 1.52 (G4(MP2)), 1.93 (G4(MP2)-6X), 1.59 (G4), and 3.81 (CBS-QB3) kcal mol ${ }^{-1}$.

\section{Conclusions}

We have investigated the $D_{5 h} \rightarrow D_{10 h}$ isomerization in the $C_{10}$ carbon cluster using the W4lite computational thermochemistry protocol. In contrast to previous high-level studies, which considered only the valence $\operatorname{CCSD}(\mathrm{T}) / \mathrm{CBS}$ reaction energy, we consider post- 
$\operatorname{CCSD}(\mathrm{T})$, core-valence, scalar relativistic, DBOC, and ZPVE corrections. We show that post-CCSD(T) and ZPVE corrections have significant contributions to the isomerization energy. In particular, our best post-CCSD(T) contributions (i.e., $\operatorname{CCSDT}(\mathrm{Q})-\operatorname{CCSD}(\mathrm{T})$ ) amount to $+2.143 \mathrm{kcal} \mathrm{mol}^{-1}$ and our best anharmonic ZPVE contribution amounts to -2.262 $\mathrm{kcal} \mathrm{mol}^{-1}$. Thus, these two dominant terms largely cancel each other out.

At the valence $\operatorname{CCSD}(\mathrm{T}) / \mathrm{cc}-\mathrm{p}\{5,6\} \mathrm{Z}$ level of theory we obtain an isomerization of +0.248 $\mathrm{kcal} \mathrm{mol}^{-1}$. There is a fair amount of cancelation between the core-valence $(-0.260)$ and scalar relativistic $\left(+0.191 \mathrm{kcal} \mathrm{mol}^{-1}\right)$ contributions. At the HF/cc-pVTZ level of theory the DBOC contribution amounts to as much as $+0.367 \mathrm{kcal} \mathrm{mol}^{-1}$, however, correlation effects at the CCSD/cc-pVTZ level of theory reduce this component to merely $+0.040 \mathrm{kcal} \mathrm{mol}^{-1}$. Overall, at the all-electron, relativistic, DBOC-inclusive, ZPVE-inclusive CCSDT(Q)/CBS level we obtain an isomerization energy of $+0.100 \mathrm{kcal} \mathrm{mol}^{-1}$.

Computationally economical composite protocols that approximate the $\operatorname{CCSD}(\mathrm{T})$ energy at $0 \mathrm{~K}$ predict that the $\mathrm{D}_{10 \mathrm{~h}}$ structure is energetically more stable than the $\mathrm{D}_{5 \mathrm{~h}}$ structure by 1.52 (G4(MP2)), 1.93 (G4(MP2)-6X), 1.59 (G4), and 3.81 (CBS-QB3) kcal mol ${ }^{-1}$.

\section{Acknowledgments}

This research was undertaken with the assistance of resources from the National Computational Infrastructure (NCI), which is supported by the Australian Government. We also acknowledge system administration support provided by the Faculty of Science at the University of Western Australia to the Linux cluster of the Karton group. We gratefully acknowledge an Australian Research Council (ARC) Future Fellowship (FT170100373). 


\section{References}

\footnotetext{
${ }^{1}$ K. W. Hinkle, J. J. Keady, P. F. Bernath, Detection of $C_{3}$ in the Circumstellar Shell of IRC+10216, Science 241, 1319 (1988).

${ }^{2}$ I. Cermak, M. Forderer, I. Cermakova, S. Kalhofer, H. Stopka-Ebeler, G. Monninger, W.
} Kratschmer, Laser-induced emission spectroscopy of matrix-isolated carbon molecules: Experimental setup and new results on $\mathrm{C}_{3}$, J. Chem. Phys. 108, 10129 (1998).

${ }^{3}$ C. Lifshitz, Carbon clusters, Int. J. Mass Spectrom. 200, 423 (2000).

${ }^{4} \mathrm{~S}$. Arulmozhiraja, T. Ohno, CCSD calculations on $\mathrm{C}_{14}, \mathrm{C}_{18}$, and $\mathrm{C}_{22}$ carbon clusters, $\mathrm{J}$. Chem. Phys. 128, 114301 (2008).

${ }^{5}$ H. W. Kroto, J. R. Heath, S. C. Obrien, R. F. Curl, R. E. Smalley, C60:

Buckminsterfullerene, Nature 318, 162 (1985).

${ }^{6}$ T. Wakabayashi, Y. Achiba, A model for the $\mathrm{C}_{60}$ and $\mathrm{C}_{70}$ growth mechanism, Chem. Phys. Lett. 190, 465 (1992).

${ }^{7}$ H. J. Dai, J. H. Hafner, A. G. Rinzler, D. T. Colbert, R. E. Smalley, Nanotubes as nanoprobes in scanning probe microscopy, Nature 384, 147 (1996).

${ }^{8}$ S. J. Tans, M. H. Devoret, H. J. Dai, A. Thess, R. E. Smalley, L. J. Geerligs, C. Dekker, Individual single-wall carbon nanotubes as quantum wires, Nature 386, 474 (1997).

${ }^{9}$ R. H. Baughman, A. A. Zakhidov, W. A. de Heer, Carbon Nanotubes - the Route Toward Applications, Science 297, 787 (2002).

${ }^{10}$ J. C. Meyer, A. K. Geim, M. I. Katsnelson, K. S. Novoselov, T. J. Booth, S. Roth, The structure of suspended graphene sheets, Nature 446, 60 (2007).

${ }^{11}$ D. P. Kosimov, A. A. Dzhurakhalov, F. M. Peeters, Carbon clusters: From ring structures to nanographene, Phys. Rev. B 81, 195414 (2010).

${ }^{12}$ A. Van Orden, R. J. Saykally, Small Carbon Clusters: Spectroscopy, Structure, and Energetics, Chem. Rev. 98, 2313 (1998). 
${ }^{13}$ K. Raghavachari, J. S. Binkley, Structure, stability, and fragmentation of small carbon clusters, J. Chem. Phys. 87, 2191 (1987).

${ }^{14}$ C. Liang, H. F. Schaefer, Carbon clusters: The structure of C10 studied with configuration interaction methods, J. Chem. Phys. 93, 8844 (1990).

${ }^{15}$ J. D. Watts, R. J. Bartlett, The nature of monocyclic $\mathrm{C}_{10}$. A theoretical investigation using coupled-cluster methods, Chem. Phys. Lett. 190, 19 (1992).

${ }^{16}$ V. Parasuk, J. Almlof, The electronic and molecular structure of carbon clusters: $\mathrm{C}_{8}$ and $\mathrm{C}_{10}$, Theor. Chim. Acta 83, 227 (1992).

${ }^{17}$ J. M. L. Martin, P. R. Taylor, Structure and Vibrations of Small Carbon Clusters from Coupled-Cluster Calculations, J. Phys. Chem. 100, 6047 (1996).

${ }^{18}$ R. O. Jones, Density functional study of carbon clusters $C_{2 n}(2 \leqslant n \leqslant 16)$. I. Structure and bonding in the neutral clusters, J. Chem. Phys. 110, 5189 (1999).

${ }^{19}$ Y. Shlyakhter, S. Sokolova, A. Lüchow, J. B. Anderson, Energetics of carbon clusters $\mathrm{C}_{8}$ and $\mathrm{C}_{10}$ from all-electron quantum Monte Carlo calculations, J. Chem. Phys. 110, 10725 (1999).

${ }^{20}$ K. Yousaf, P. R. Taylor, On the electronic structure of small cyclic carbon clusters, Chem. Phys. 349, 58 (2008).

${ }^{21}$ A. Karton, J. M. L. Martin, Atomization energies of the carbon clusters Cn $(n=2-10)$ revisited by means of W4 theory as well as density functional, Gn, and CBS methods, Mol. Phys. 107, 977 (2009).

${ }^{22}$ T. W. Yen, S. K. Lai, Use of density functional theory method to calculate structures of neutral carbon clusters $C_{n}(3 \leq n \leq 24)$ and study their variability of structural forms, J. Chem. Phys. 142, 084313 (2015). 
${ }^{23}$ Z.-Q. Wang, C.-E. Hu, X.-R. Chen, Y. Cheng, Q.-F. Chen, Ab initio investigation of structure, spectrum, aromaticity and electronic properties of $\mathrm{C}_{10}$ carbon cluster, Comput. Theor. Chem. 1118, 94 (2017).

${ }^{24}$ A. Karton, E. Rabinovich, J. M. L. Martin, B. Ruscic, W4 theory for computational thermochemistry: In pursuit of confident sub-kJ/mol predictions, J. Chem. Phys. 125, 144108 (2006).

${ }^{25}$ A. Karton, A computational chemist's guide to accurate thermochemistry for organic molecules, WIREs Comput. Mol. Sci. 6, 292 (2016).

${ }^{26}$ A. Karton, S. Daon, J. M. L. Martin, W4-11: A high-confidence benchmark dataset for computational thermochemistry derived from first-principles W4 data, Chem. Phys. Lett. 510,165 (2011).

${ }^{27}$ A. Karton, I. Kaminker, J.M.L. Martin, Economical Post-CCSD(T) Computational Thermochemistry Protocol and Applications to Some Aromatic Compounds, J. Phys. Chem. A $113,7610(2009)$.

${ }^{28}$ H.-J. Werner, P. J. Knowles, G. Knizia, F. R. Manby, M. Schutz, P. Celani, T. Korona, R. Lindh, A. Mitrushenkov, G. Rauhut, K. R. Shamasundar, T. B. Adler, R. D. Amos, A. Bernhardsson, A. Berning, D. L. Cooper, M. J. O. Deegan, A. J. Dobbyn, F. Eckert, E. Goll, C. Hampel, A. Hesselmann, G. Hetzer, T. Hrenar, G. Jansen, C. Koppl, Y. Liu, A. W. Lloyd, R. A. Mata, A. J. May, S. J. McNicholas, W. Meyer, M. E. Mura, A. Nickla, D. P. O’Neill, P. Palmieri, D. Peng, K. Pfluger, R. Pitzer, M. Reiher, T. Shiozaki, H. Stoll, A. J. Stone, R. Tarroni, T. Thorsteinsson, M. Wang, MOLPRO is a package of ab initio programs; available at: http:www. molpro.net.

${ }^{29}$ H.-J. Werner, P. J. Knowles, G. Knizia, F. R. Manby, M. Schutz, Molpro: a generalpurpose quantum chemistry program package, WIREs Comput. Mol. Sci. 2, 242 (2012). 
${ }^{30}$ M. Kallay, Z. Rolik, J. Csontos, P. Nagy, G. Samu, D. Mester, I. Ladjanszki, L. Szegedy, B. Ladoczki, K. Petrov, M. Farkas, B. Hegely. MRCC: A quantum chemical program suite. See also: http://www.mrcc.hu.

${ }^{31}$ Z. Rolik, L. Szegedy, I. Ladjanszki, B. Ladoczki, M. Kallay, An efficient linear-scaling CCSD(T) method based on local natural orbitals, J. Chem. Phys. 139, 094105 (2013).

${ }^{32}$ J. F. Stanton, J. Gauss, M. E. Harding, P. G. Szalay with contributions from A. A. Auer, R. J. Bartlett, U. Benedikt, C. Berger, D. E. Bernholdt, Y. J. Bomble, L. Cheng, O. Christiansen, M. Heckert, O. Heun, C. Huber, T.-C. Jagau, D. Jonsson, J. Juselius, K. Klein, W. J. Lauderdale, D. A. Matthews, T. Metzroth, L. A. Muck, D. P. O’Neill, D. R. Price, E. Prochnow, C. Puzzarini, K. Ruud, F. Schiffmann, W. Schwalbach, C. Simmons, S. Stopkowicz, A. Tajti, J. Vazquez, F. Wang, J. D. Watts and the integral packages MOLECULE (J. Almlof, P. R. Taylor), PROPS (P. R. Taylor), ABACUS (T. Helgaker, H. J. Aa. Jensen, P. Jørgensen, J. Olsen), and ECP routines by A. V. Mitin, C. van Wullen. CFOUR, a Quantum Chemical Program Package; For the current version, see http://www. cfour.de.

${ }^{33}$ M. J. Frisch, G. W. Trucks, H. B. Schlegel, G. E. Scuseria, M. A. Robb, J. R. Cheeseman, G. Scalmani, V. Barone, B. Mennucci, G. A. Petersson, et al. Gaussian 09, Revision D.01; Gaussian, Inc.: Wallingford CT, 2009.

${ }^{34}$ T. H. Dunning, Gaussian basis sets for use in correlated molecular calculations. I. The atoms boron through neon and hydrogen, J. Chem. Phys. 90, 1007 (1989).

${ }^{35}$ K. A. Peterson, T. H. Dunning, Accurate correlation consistent basis sets for molecular core-valence correlation effects: The second row atoms $\mathrm{Al}-\mathrm{Ar}$, and the first row atoms $\mathrm{B}-\mathrm{Ne}$ revisited, J. Chem. Phys. 117, 10548 (2002). 
${ }^{36}$ W.A. de Jong, R.J. Harrison, and D.A. Dixon, Parallel Douglas-Kroll energy and gradients in NWChem: Estimating scalar relativistic effects using Douglas-Kroll contracted basis sets, J. Chem. Phys. 114, 48 (2001).

${ }^{37}$ A. Karton, J. M. L. Martin, Comment on: "Estimating the Hartree-Fock limit from finite basis set calculations" [Jensen F (2005) Theor Chem Acc 113:267], Theor. Chem. Acc. 115, 330 (2006).

${ }^{38}$ F. Jensen, Estimating the Hartree-Fock limit from finite basis set calculations, Theor. Chem. Acc. 113, 267 (2005).

${ }^{39}$ A. Karton, P. R. Taylor, J. M. L. Martin, Basis set convergence of post-CCSD contributions to molecular atomization energies, J. Chem. Phys. 127, 064104 (2007).

${ }^{40}$ N. Sylvetsky, K. A. Peterson, A. Karton, J. M. L. Martin, Toward a W4-F12 approach: Can explicitly correlated and orbital-based ab initio $\operatorname{CCSD}(\mathrm{T})$ limits be reconciled?, J. Chem. Phys. 144, 214101 (2016).

41 J. M. L. Martin, G. de Oliveira, Towards standard methods for benchmark quality ab initio thermochemistry - W1 and W2 theory, J. Chem. Phys. 111, 1843 (1999).

${ }^{42}$ S. Parthiban, J. M. L. Martin, Assessment of W1 and W2 theories for the computation of electron affinities, ionization potentials, heats of formation, and proton affinities, J. Chem. Phys. 114, 6014 (2001).

${ }^{43}$ A. Karton, P. R. Schreiner, J. M. L. Martin, Heats of Formation of Platonic Hydrocarbon Cages by Means of High-Level Thermochemical Procedures, J. Comput. Chem. 37, 49 (2016).

${ }^{44}$ M. K. Kesharwani, B. Brauer, J. M. L. Martin, Frequency and Zero-Point Vibrational Energy Scale Factors for Double-Hybrid Density Functionals (and Other Selected Methods): Can Anharmonic Force Fields Be Avoided?, J. Phys. Chem. A 119, 1701 (2015). 
${ }^{45}$ A. D. Becke, Density-functional thermochemistry. III. The role of exact exchange, J. Chem. Phys. 98, 5648 (1993).

${ }^{46}$ F. Jensen, Polarization consistent basis sets: Principles, J. Chem. Phys. 115, 9113 (2001).

${ }^{47}$ B. Ruscic, R. E. Pinzon, M. L. Morton, G. von Laszewski, S. J. Bittner, S. G. Nijsure, K. A. Amin, M. Minkoff, A. F. Wagner, Introduction to Active Thermochemical Tables: Several "Key” Enthalpies of Formation Revisited, J. Phys. Chem. A 108, 9979 (2004).

${ }^{48}$ L. A. Curtiss, P. C. Redfern, K. Raghavachari, Gn Theory, WIREs Comput. Mol. Sci. 1, $810(2011)$.

${ }^{49}$ B. Chan, J. Deng, L. Radom, G4(MP2)-6X: A Cost-Effective Improvement to G4(MP2), J. Chem. Theory Comput. 7, 112 (2011).

${ }^{50}$ J. A. Montgomery Jr, M. J. Frisch, J. W. Ochterski, G. A. Petersson, A complete basis set model chemistry. VI. Use of density functional geometries and frequencies, J. Chem. Phys. 110, 2822 (1999). 